Received: 2015.11.22

Accepted: 2015.12 .14

Published: 2016.07.07

\title{
A Technical Perspective for Understanding Quantitative Arterial Spin-Labeling MR Imaging Using Continuous ASL
}

\author{
Tomoyuki Noguchi ${ }^{\text {ABCDEFE }}$
}

Department of Radiology, National Center for Global Health and Medicine, Toyama, Shinjuku-ku, Tokyo, Japan

Author's address: Tomoyuki Noguchi, Department of Radiology, National Center for Global Health and Medicine, 1-21-1, Toyama, Shinjuku-ku, Tokyo, 162-8655, Japan, e-mail: tnogucci@radiol.med.kyushu-u.ac.jp

Source of support: Grant-in-Aid for Scientific Research of Japan Society for the Promotion of Science

\section{Summary}

The current paper describes visually the system of CBF measurement by continuous ASL using schematic illustration. I also discussed the effects of the parameters used in continuous ASL to CBF values as measured with ASL-MRI.

MeSH Keywords:

Brain • Magnetic Resonance Imaging • Models, Theoretical • Perfusion Imaging • Spin Labels

PDF file: http://www.polradiol.com/abstract/index/idArt/896795

\section{Background}

Arterial spin-labeling magnetic resonance imaging (ASLMRI) is a non-invasive imaging method to evaluate brain perfusion [1,2]. ASL-MRI requires no contrast media or any other extrinsic tracer administration because it uses the blood water molecule as an intrinsic tracer. In brief, ASL-MRI initially applies inversion radiofrequency pulses proximal to the brain and then images the brain where the arterial blood with the inverted magnetization perfuses into the brain microvasculature. To accomplish this scheme, ASL-MRI includes 2 essential components of the MRI sequences: the spin-labeling sequence and the image acquisition sequence [1,2]. These sequences are combined and used for obtaining three sets of images; the reference images, the labeled images, and the control images. At the first step, the reference images of the brain are taken with the imaging data acquisition sequence. At the second step, the labeled images are acquired using both the spin-labeling and the image acquisition sequences with the following methods: 1) applying an inversion pulse at the cervical area and labeling the arterial blood just before travelling into the brain by inverting the spin of the hydrogen nucleus of the water molecules; 2) waiting for the spin-labeled arterial blood to perfuse throughout the brain (post labeling delay, or PLD) [3], 3) performing the sequence of the imaging data acquisition which takes images of the brain. At the third step, the control images are obtained with the same process used in taking the labeled images after the unlabeled arterial blood perfuses in the brain. In fact, the labeled images exhibit only slightly lower signal values than control images depending on the perfusion rate of labeled arterial blood. To extract this tiny difference, the subtraction images, which will represent cerebral blood flow (CBF)-weighted images, are calculated by subtracting the labeled images from the control images. Finally, the CBF maps are generated from the subtraction images calibrated by the signal intensity of the arterial blood with full relaxed magnetization per unite measured from the reference images.

ASL-MRI can be broadly divided into two types according to the spin-labeling technique used. These are continuous ASL (CASL) [1-6] and pulsed ASL (PASL) [7-13]. CASL involves repeatedly applying an inversion pulse over a small area and continuously labeling the arterial blood that flows into that area, whereas PASL involves applying an inversion pulse over a relatively wide area and labeling all of the arterial blood in that area at once.

CASL has the advantage of having a high signal to noise ratio (SNR) compared with PASL (theoretically, approximately 2.71 times that of PASL $[10,14])$. As a general rule, $\mathrm{CBF}$ is approximately $60 \mathrm{~mL} / 100 \mathrm{~g} / \mathrm{min}$, which means that in 1 second, $1 \mathrm{~mL}$ of arterial blood perfuses into the brain tissue; even if roughly calculated, it is only $1 \%$ of 
the signal intensity of the brain tissue. In this respect, a high SNR is important. On the other hand, applying a continuous inversion pulse is a technique that exhibits a high specific absorption rate (SAR), and may be technically difficult for the hardware to perform. In particular, concerns have arisen regarding patient safety at 3.0 Tesla. However, recent developments in pseudo-continuous ASL (pCASL) [4], a technique in which an intermittent inversion pulse is applied at regular intervals, has enabled its clinical application with 3.0-Tesla MRI devices, and it is expected that it can be used in future with 7.0-Tesla ultra-high magnetic resonance devices. Furthermore, commercial versions of 3D ASL [5] have become available, combining imaging technologies such as the 3D-spiral FSE method, which acquires images of the whole brain, as well as the background suppression method that suppresses signals from the stationary tissue unrelated to perfusion [15]. While we will leave the details of these technologies to other papers and handbooks, we will briefly explain the equations to calculate absolute CBF in continuous ASL in the present paper.

\section{Quantitative equation of cerebral blood flow by continuous ASL}

CASL developed by Detre was the original ASL model [1]. To begin with, they performed a study using rat subjects; thereafter, they added improvements such as including a delay period in the sequence following spin-labeling and attempting to quantify $\mathrm{CBF}$ in humans [3]. Dai et al. proposed a sequence to acquire images in CASL, namely pCASL [4], and they succeeded in overcoming the aforementioned technological limitations associated with applying a continuous inversion pulse. Other subsequent improvements were added and a commercial version of 3D ASL was launched. Here we will explain the system of CBF measurement by continuous ASL proposed by Järnum et al. using the equation below [5].

$$
f=\frac{\lambda}{2 \alpha T_{1 b}\left(1-e^{-\tau / T 1 b}\right)} \frac{\left(S_{c t r l}-S_{l b l}\right)\left(1-e^{-t_{s a t} / T_{1 g}}\right)}{S_{\text {ref }}} e^{w / T 1 b}
$$

Here $f$ refers to $\mathrm{CBF}$, and $S_{c t r l}, S_{l b l}$, and $S_{\text {ref }}$ refer to the signal intensity of the control images, label images, and reference images, respectively; $T_{l b}$ and $T_{l g}$ refer to the $\mathrm{Tl}$ values of the arterial blood and grey matter, respectively, $\alpha$ refers to spin labeling efficiency, $\lambda$ is the brain-blood coefficient of the water, $\tau$ is the duration of the labeling application, $w$ is the PLD, and $t_{\text {sat }}$ is the time of water suppression pulse application used to acquire the reference images.

To help understand this equation, a schematic diagram will be used (Figure 1). One gram of a brain tissue block has one arterial branch passing through its center. The longitudinal magnetization of $1 \mathrm{~mL}$ of the arterial blood within the blood vessel is $\mathrm{M}_{0 \mathrm{~B}}$, and inside the blood vessel, arterial blood flows at a blood flow volume $f(\mathrm{~mL} / \mathrm{g} / \mathrm{s})$ (Figure 1A). Next, the labeling slab is set below the brain tissue block, i.e., upstream of the arterial blood and the inversion pulse is initiated (elapsed time $=0 \mathrm{~s}$ ) (Figure 1B). Thereafter, once the inversion pulse is applied continuously for $\tau$ seconds (Figure 1C) arterial blood flows at a blood flow volume $f$, resulting in a total amount of labeled blood after elapsed time $\tau$ of $f \cdot \tau(\mathrm{mL})$. After the labeled blood is inverted, it is recovered with the arterial blood $\mathrm{Tl}$ value $\left(T_{1 b}\right)$. As a result, the micro-volume of arterial blood $f \cdot d t(\mathrm{~mL})$ flowing from the labeled region at a very short time immediately after starting labeling has a magnetization intensity of $\mathrm{M}_{0 \mathrm{~B}}\left(1-2 \mathrm{e}^{-\tau / \mathrm{Tl}}\right)$ after $\tau$ seconds. Next micro-volume of arterial blood $f \cdot d t$ $(\mathrm{mL})$ flowing after a short time of $2 \times d t$ seconds of starting labeling has a magnetization intensity of $\mathrm{M}_{0 b}\left(1-2 \mathrm{e}^{-(\tau-d t) / \mathrm{Tl} b}\right)$. Another micro-volume of arterial blood $f \cdot d t(\mathrm{~mL})$ after $3 \times d t$ seconds has a magnetization intensity of $\mathrm{M}_{0 b}\left(1-2 \mathrm{e}^{-(\tau-2 d t / / \mathrm{Tl} l b}\right)$. Therefore, it has a magnetization intensity of $\mathrm{M}_{0 \mathrm{~b}}\left(1-2 \mathrm{e}^{-d t /}\right.$ ${ }^{\mathrm{Tlb}}$ ) immediately before completion of labeling.

Thereafter, one waits until this labeled blood reaches and perfuses brain tissue (several background signal suppression pulses as well as several inferior saturation pulses to suppress inflowing arterial blood spins after labeling are applied during this delay period [5]). The labeled images are taken after $(\tau+w)$ seconds have elapsed. At this time, the overall volume of magnetization intensity of all labeled blood is $\int_{+w}^{\tau+w} f \cdot M_{0 b}\left(1-2 e^{-t / T 1 b}\right) d t$ (Figure 1D). Based on the indefinite integral $\int e^{-x / 4} d x=-A e^{-x / A}+C$, it follows that:

$$
\int_{w}^{\tau+w} f \cdot M_{0 b}\left(1-2 e^{-t / 71 b}\right) d t=f \cdot M_{0 b}\left[t-\left(-2 T_{1 b} e^{-t / T T_{b} b}\right]_{w}^{[+w}=f \cdot M_{0 b}\left(\tau-\frac{2 T_{1 b}\left(1-e^{-\tau / T / 1 b b}\right)}{e^{w / T / b}}\right)\right.
$$

The control images with double inversion pulse application are taken simultaneously (Figure 1E). At this time, the overall volume of magnetization intensity of unlabeled arterial blood is $M_{0 b} \cdot f \cdot \tau$. Accordingly, the measured longitudinal magnetization difference, $\Delta M$, is acquired by subtracting labeled images from control images as shown below (Figure $1 \mathrm{~F}$ ).

$$
\Delta M=f \cdot M_{0 b} \cdot \tau-f \cdot M_{0 b}\left(\tau-\frac{2 T_{1 b}\left(1-e^{-\tau / T 1 b}\right)}{e^{w / T 1 b}}\right)=\frac{2 T_{1 b}\left(1-e^{-\tau / T 1 b}\right)}{e^{w / T I b}} \cdot f \cdot M_{0 b}
$$

However, in actual practice, since the rate of spin labeling by inversion pulse is not $100 \%, \Delta M$ is lower than the theoretical value of $\frac{2 T_{1 b}\left(1-e^{-t / 71 b}\right)}{e^{w / T / b}} \cdot f \cdot M_{0 b}$. To correct this, the spin labeling rate is multiplied by $\alpha$.

$$
\Delta M=\frac{2 \alpha T_{1 b}\left(1-e^{-\tau / T 1 b}\right)}{e^{w / T 1 b}} \cdot f \cdot M_{0 b}
$$

$\Delta M$ on the left side of equation (4) is calculated from the difference in signal intensity values between the control and labeled images $\left(S_{c t r l}-S_{l b l}\right)$. If the repetition time is TR, the effective echo time is $\mathrm{TE}$, and the $\mathrm{T} 2$ value of the grey matter is $T_{2 g}$, the equation is as follows:

$$
\begin{aligned}
& S_{c t r l}-S_{r e f}=\Delta M \cdot\left(1-e^{-T R / T_{1 g}}\right) e^{-T E / T_{2 g}} \\
& \leftrightarrow \Delta M=\frac{S_{c t r l}-S_{l b l}}{\left(1-e^{-T R / T_{1 g}}\right) e^{-T E / T_{2 g}}}
\end{aligned}
$$

On the other hand, $M_{0}$ included on the right side of the equation (4) uses $\lambda$ the brain tissue-blood coefficient for water. In other words, when measuring blood flow in living organisms, it is hypothesized that NMR signals responsible for blood flow are mainly derived from the water molecule $\mathrm{H}_{2} \mathrm{O}$ [2]. Therefore, if the brain tissue magnetization intensity is $M_{0}$, the following equation can be concluded:

$$
\begin{aligned}
& \lambda=\frac{\text { tissue water content }}{\text { blood water content }}=\frac{M_{0}}{M_{0 b}} \\
& \leftrightarrow M_{o b}=\frac{M_{0}}{\lambda}
\end{aligned}
$$



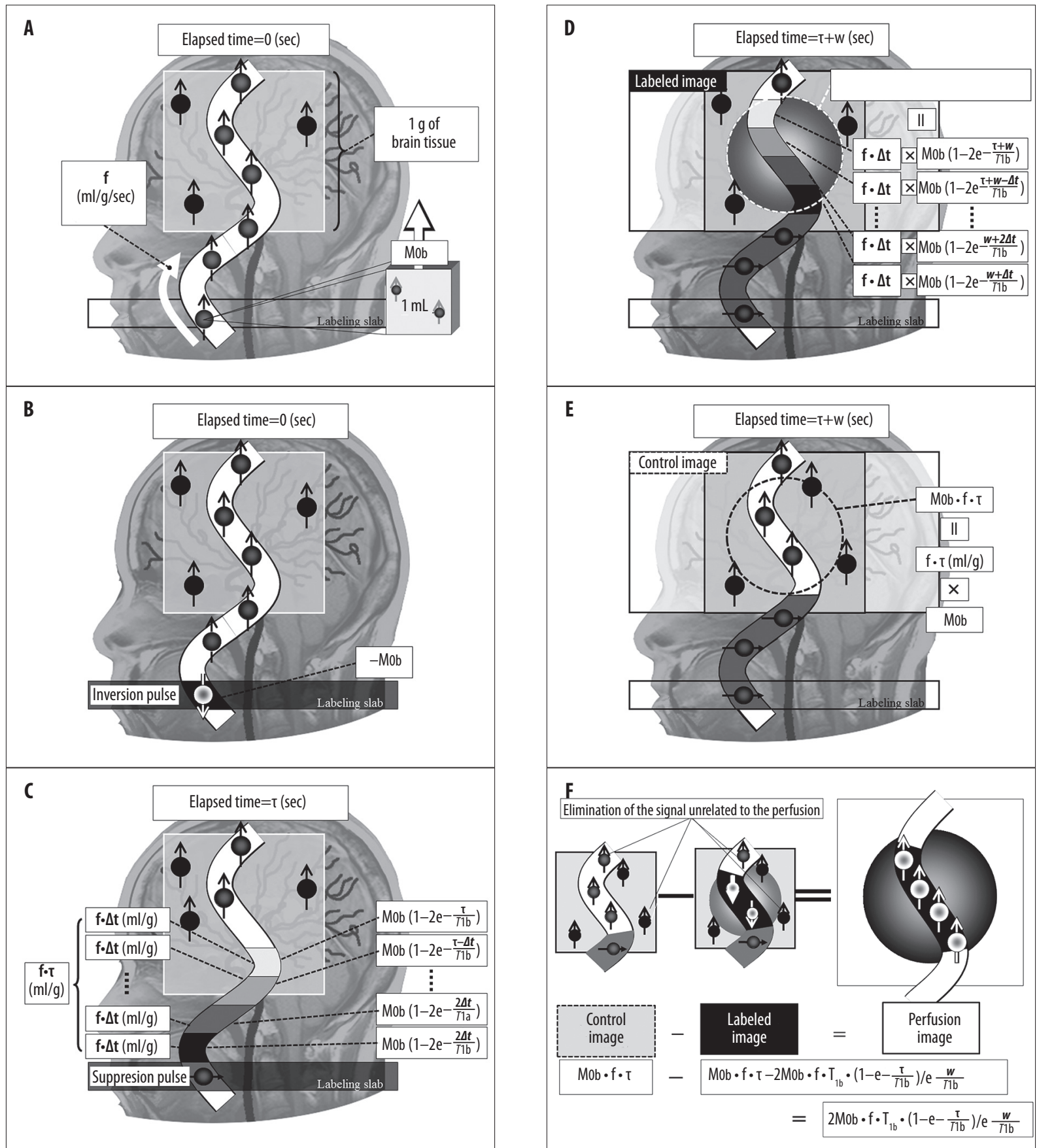

Figure 1. Schematic diagram to explain continuous ASL blood flow quantification. (A) In $1 \mathrm{~g}$ of a brain tissue block, the magnetization intensity of $1 \mathrm{~mL}$ of intravascular arterial blood is $M_{0 B}$. Intravascular arterial blood is therefore hypothesized as flowing at a blood flow volume of $f(\mathrm{~mL} / \mathrm{g} / \mathrm{s})$. (B) The elapsed time when the continuous inversion pulse has been applied to the labeling slab is set at 0 . (C) After elapsed time of $\mathrm{s} \mathrm{s}$, one waits until completion of the continuous inversion pulse on the labeling slab (the several selective and nonselective saturation pulses are applied during this time). (apacity of labeled blood is $f \cdot \tau(\mathrm{mL})$. Furthermore, as the labeled blood is recovered with $T_{1 b}$, the arterial blood T1 value, magnetization intensity per $1 \mathrm{~mL}$ after inversion pulse application is expressed as $-M_{0 b}\left(1-2 e^{-t / T 1 b}\right)$. (D) The labeled images are taken after elapsed time of $(\tau+W) \mathrm{sec}$. At this time, the total volume of magnetization intensity of labeled blood is $\int_{\mathrm{w}}{ }^{\mathrm{T}+\mathrm{w}} \mathrm{f} \cdot M_{0 b} \cdot\left(1-2 \mathrm{e}^{-t / 71 b}\right) d t$. (E) Next, control images to which the inversion pulse has not been applied are taken at the same time. At this time, the total volume of magnetization intensity of the unlabeled arterial blood is $M_{0 b} \cdot f \cdot \tau$. (F) For the actual signal difference, $\Delta M$ obtained by subtracting label images from control images, $2 M_{0 b} \cdot f \cdot T_{1 b} \cdot\left(1-e^{-\tau / T 1 b)} / e^{w / T 1 b}\right.$, is acquired by subtracting $M_{0 b} \cdot f \cdot\left\{\tau-2 T_{1 b}\left(1-e^{-\tau / T 1 b}\right) /\right.$ $\left.e^{w / T h}\right\}$ from $M_{O B} \cdot f \cdot \tau$. 
Proton-density-weighted images (PD images) are used for $M_{0}$ measurement. However, the receivable signal range may be exceeded when standard PD images are used [16]. Therefore, the reference images are used with the same $\mathrm{TE}$ and TR as the water suppression PD images that apply a saturation pulse $t_{\text {sat }}$ seconds before acquiring PD images [16] (these reference images are also called water sensitivity images [17]). Therefore, $M_{0}$ may be expressed as follows using the signal intensity obtained:

$$
\begin{aligned}
& S_{r e f}=M_{0} \cdot\left(1-e^{-T R / T_{1 g}}\right) e^{-T E / T_{2 g}} \cdot\left(1-e^{-t_{s a t} / T_{1 g}}\right) \\
& \Leftrightarrow M_{0}=\frac{S_{r e f}}{\left(1-e^{-T R / T_{1 g}}\right) e^{-T E / T_{2 g}} \cdot\left(1-e^{-t_{s a t} / T_{1 g}}\right)}
\end{aligned}
$$

Therefore, when (5) is substituted into the left side of equation (4), with (6) and (7) into the right side, it creates equation (1) for $f$.

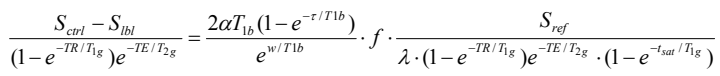

$$
\begin{aligned}
& \leftrightarrow S_{c r t l}-S_{l b l}=\frac{2 \alpha T_{1 b}\left(1-e^{-\tau / T 1 b}\right)}{e^{w / I l b}} \cdot f \cdot \frac{S_{r e f}}{\lambda \cdot\left(1-e^{-t_{s a t} / T_{g}}\right)} \quad-(8) \\
& \leftrightarrow f=\frac{\lambda}{2 \alpha T_{1 b}\left(1-e^{-\tau / T 1 b}\right)} \frac{\left(S_{c r r l}-S_{l b l}\right)\left(1-e^{-t_{s a r} / T_{\mathrm{Ig}}}\right)}{S_{r e f}} e^{w / T 1 b} \quad-(1)
\end{aligned}
$$

However, equation (1) is only true when $\tau<\tau_{\max }$ or $\Delta t<w$. $\tau_{\max }$ is the maximum continued time of label application up to when the labeled arterial blood within the brain tissue becomes fully inverted and there is no further improvement in SNR on the subtraction images. $\Delta t$ is the delay for labeled blood to first arrive at the imaging area after application of the inversion pulse. Other conditions are expressed as below [14]:

$$
\begin{array}{rr}
\Delta \mathrm{M}=0 & (w<\Delta t-\tau) \\
\Delta M=\frac{2 \alpha T_{1 b}\left(e^{-(\Delta t-w) / T 1 b}-e^{-\tau / T 1 b}\right)}{e^{w / T 1 b}} \cdot f \cdot M_{0 b}(\Delta t-\tau<w<\Delta t) \\
\Delta M=\frac{2 \alpha T_{1 b}\left(1-e^{-\tau / T 1 b}\right)}{\rho^{w / T 1 b}} \cdot f \cdot M_{0 b} & \left(\Delta t<w \text { and } \tau<\tau_{\max }\right) \quad(11)(0 r \\
\Delta M=\frac{2 \alpha T_{1 b}\left(1-e^{-\tau \max / T 1 b}\right)}{e^{w / T 1 b}} \cdot f \cdot M_{0 b} & \left(\Delta t<w \text { and } \tau_{\max }<\tau\right)
\end{array}
$$

\section{Continuous ASL in finished product version}

Equations (9) to (12) reflect changes in $w$ at a gradual increase from 0 when blood flow $f$ is constant. Accordingly, $w$ must be set after ascertaining $\Delta t$ of each area of the targeted brain. However, $\Delta t$ varies according to differences in patient age, measurement site, presence or absence of a lesion narrowing or blocking the artery, and anomalous arteries. Moreover, $\Delta t$ may be influenced by blood flow $f$. Furthermore, when $w$ is prolonged too much, the inversion spin of the labeled blood returns to normal, which may reduce the signal difference between control and labeled images and therefore the SNR as well. Thus, it is not easy to set $w$ according to each individual.
In actual clinical settings in the finished version, $w$ is not set for each of the individual cases but used as a fixed value. For example, in the event of clinical 3.0-Tesla MRI devices, $W$ is often fixed at either 1.5 seconds or 2.5 seconds. Under such practical conditions, CBF images may be sensitive to the reduced blood flow or blood flow velocity, such as in cerebral infarction, cerebrovascular stenosis, and obstruction. In this section, the imaging phantom is assumed to be a single, well-mixed compartment with intra- and extravascular water in perfect communication with penetration of a single tube, similar to the model shown in Figure 1. Labeled water enters and leaves with perfusion rate $f$. These conditions are specifically expressed in the following ways:

$$
\begin{array}{lr}
f^{\prime}=0 & \left(0<f<f_{\text {min }}\right) \\
f^{\prime}=\frac{e^{(1-f \min / f) \cdot(\tau+w) / T 1 b}-1}{e^{\tau / T 1 b}-1} \cdot f & \left(f_{\text {min }}<f<\frac{\tau+w}{w} \cdot f_{\text {min }}\right) \\
f^{\prime}=f & \left(\frac{\tau+w}{w} \cdot f_{\text {min }}<f<f_{\text {peak }}\right) \\
f^{\prime}=f_{\text {peak }} & \left(f_{\text {peak }}<f\right)
\end{array}
$$

Here, $f^{\prime}$ expresses CBF value measured with ASL while $f$ signifies true $\mathrm{CBF}$ value. To explain equation (13), $f_{\min }$ is the minimum $\mathrm{CBF}$ value that can be detected from the signal difference $\Delta M$ between control images and label images with given fixed $\tau$ and $w$. Therefore, it follows that as long as $f$ does not reach fmin, $f^{\prime}$ cannot be detected with ASL and remains 0 . Moreover, if $v_{i a}$ is set as the virtual arterial vessel internal volume of the path that labeled blood is flowing through until reaching the region of interest, the equation $v_{\mathrm{ia}}=(\tau+w) \cdot f_{\min }$ is established. Next, equation (14) can be explained in the following manner. Even if CBF value increases more than $f_{\text {min }}, f^{\prime}$ is underestimated more than $f$. This is because equation (11) is practically used for calculation of $f^{\prime}$ although equation (10) should be theoretically used. Therefore, it follows that:

$$
\begin{aligned}
& \frac{2 \alpha T_{1 b}\left(e^{-(\Delta t-w) / T 1 b}-e^{-\tau / T 1 b}\right)}{e^{w / T 1 b}} \cdot f \cdot M_{0 b}=\frac{2 \alpha T_{1 b}\left(1-e^{-\tau / T 1 b}\right)}{e^{w / T 1 b}} \cdot f^{\prime} \cdot M_{0 b} \\
& \Leftrightarrow f^{\prime}=\frac{e^{(\tau+w-\Delta t) / T 1 b}-1}{e^{\tau / T 1 b}-1} \cdot f
\end{aligned}
$$

Meanwhile, when CBF value $f$ increases, $\Delta t$ decreases. However, the labeled blood follows the same path. Therefore:

$$
\begin{aligned}
& v_{i a}=(\tau+w) \cdot f_{\text {min }}=\Delta t \cdot f \\
& \leftrightarrow \Delta t=\frac{f_{\text {min }}}{f}(\tau+w)
\end{aligned}
$$

Substituting this creates equation (14). When $f$ increases further, equation (11) (i.e., equation (4)) is finally valid for $f^{\prime}$. Therefore, equation (15), $f^{\prime}=f$, is established. The condition for this is a state of $\frac{\tau+w}{w} \cdot f_{\min }<f$. However, this is when $f$ is substituted for the $f^{\prime \prime}$ in equation (14). Furthermore, when $\Delta M$ eventually stops changing even with increases in $f, f^{\prime}$ becomes fixed at a specific blood flow value of $f_{\text {peak }}$. This is because the labeled blood volume in the brain tissue has reached its maximum. If this maximum blood volume is expressed as $v_{\text {label, }}$, then $v_{\text {label }}=f_{\text {peak }} \cdot \tau$ is true. If the duration of the pseudo-continuous inversion pulse can be infinitely extended, the CBV $(\mathrm{mL} / \mathrm{lg}$ brain tissue $)=f_{\text {peak }} \cdot \tau$. 


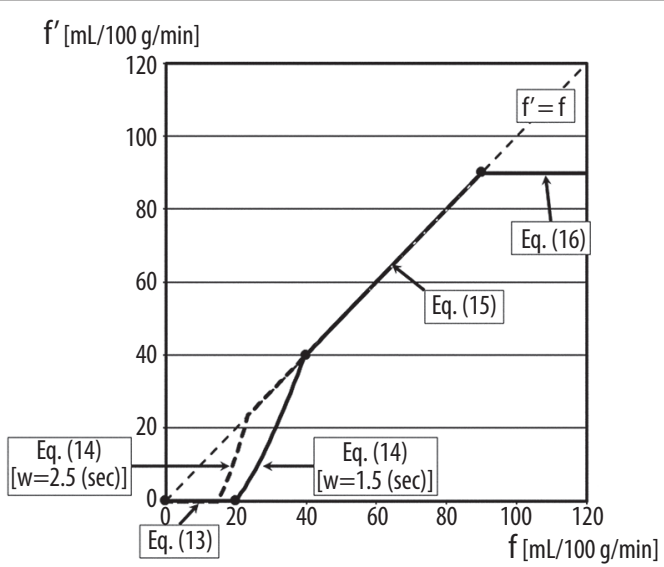

Figure 2. The virtual graph showing the relationship between true $\mathrm{CBF}$ value and CBF value as measured with ASL with fixed $\tau$ and $w$. In this graph, $f^{\prime}$ is CBF value measured with $A S L$, $f$ is true CBF value, $\tau$ is $1.5 \mathrm{sec}$, $w$ is $1.5 \mathrm{sec}$ (continuous line) and $2.5 \mathrm{sec}$ (dashed line), $f_{\min }$ is $20 \mathrm{~mL} / 100 \mathrm{~g} / \mathrm{min}$ and $f_{\text {peak }}$ is $90 \mathrm{~mL} / 100$ $\mathrm{g} / \mathrm{min}$. Equation (13) shows that since the true CBF value is low it cannot be detected with ASL and f' is zero. Equation (14) shows that $f^{\prime}$ can be measured with ASL when fincreases, but it is lower than true blood flow value and describes a concave down curve. However, when $f$ increases further, equation (15), $f^{\prime}=f$, applies. Accordingly, when $\Delta M$ eventually stops changing even with increase in $f$, $f^{\prime}$ becomes fixed at a specific blood flow value of $f_{\text {peak. }}$. This is equation (16).

Figure 2 depicts a virtual graph of (13) to (16) with $f_{\min }=20$ $(\mathrm{mL} / 100 \mathrm{~g} / \mathrm{min})$, fpeak $=90(\mathrm{~mL} / 100 \mathrm{~g} / \mathrm{min}), \tau=1.5(\mathrm{sec})$, $w=1.5$ (sec) and $w=2.5$ (sec), particularly to highlight changes in equation (14). In equation (14) with $w=1.5$ (sec), the approximated curve using the secondary equation is:

$$
f^{\prime}=0.0323 f^{2}+0.086 f-14.854 \quad\left(\mathrm{r}^{2}=0.9999\right)
$$

Since equation (19) is a concave down parabola, it means that equation (14) draws a curve that is slightly concave down compared to a straight line. Moreover, a condition of equation (19) is that $w=1.5(\mathrm{sec})$ and that $f$ is in the range of $20-40(\mathrm{~mL} / 100 \mathrm{~g} / \mathrm{min})$; but when $w=2.5(\mathrm{sec}), f$ changes to $15-24(\mathrm{~mL} / 100 \mathrm{~g} / \mathrm{min})$.

However, the equations (13) to (16) do not necessarily represent the actual situation of the human brain perfusion because the normal cerebral blood passes through multiple paths and the reduced blood supply can lead to development of the collateral channels. It should be emphasized again that these equations have been simplified in order to roughly understand the differences in cerebral blood flow values and true cerebral blood flow value measured in ASL-MRI.

\section{Conclusions}

Although ASL is a useful MRI technique that allows for CBF to be measured non-invasively, low SNR has been a problem. Continuous ASL can provide relatively reliable measurements with a high SNR, and we expect that it will gain further popularity in future. We hope that this paper helps in understanding of equations to measure absolute cerebral blood flow and to gain deeper insight into the mechanisms underlying continuous ASL.

\section{Conflicts of interest}

Author confirm that there are no known conflicts of interest associated with this publication and there has been no significant financial support for this work that could have influenced its outcome.

\section{References:}

1. Detre JA, Leigh JS, Williams DS, Koretsky AP: Perfusion imaging. Magn Reson Med, 1992; 23(1): 37-45

2. Williams DS, Detre JA, Leigh JS, Koretsky AP: Magnetic resonance imaging of perfusion using spin inversion of arterial water. Proc Natl Acad Sci USA, 1992; 89(1): 212-16

3. Alsop DC, Detre JA: Reduced transit-time sensitivity in noninvasive magnetic resonance imaging of human cerebral blood flow. J Cereb Blood Flow Metab, 1996; 16(6): 1236-49

4. Dai W, Garcia D, de Bazelaire C, Alsop DC: Continuous flow-driven inversion for arterial spin labeling using pulsed radio frequency and gradient fields. Magn Reson Med, 2008; 60(6): 1488-97

5. Jarnum H, Steffensen EG, Knutsson L et al: Perfusion MRI of brain tumours: a comparative study of pseudo-continuous arterial spin labelling and dynamic susceptibility contrast imaging. Neuroradiology, 2010; 52(4): 307-17

6. Alsop DC, Detre JA: Multisection cerebral blood flow MR imaging with continuous arterial spin labeling. Radiology, 1998; 208(2): 410-16

7. Kwong KK, Belliveau JW, Chesler DA et al: Dynamic magnetic resonance imaging of human brain activity during primary sensory stimulation. Proc Natl Acad Sci USA, 1992; 89(12): 5675-79

8. Edelman RR, Siewert B, Darby DG et al: Qualitative mapping of cerebral blood flow and functional localization with echo-planar MR imaging and signal targeting with alternating radio frequency. Radiology, 1994; 192(2): 513-20

9. Wong EC, Buxton RB, Frank LR: Implementation of quantitative perfusion imaging techniques for functional brain mapping using pulsed arterial spin labeling. NMR Biomed, 1997; 10(4-5): 237-49
10. Wong EC, Buxton RB, Frank LR: Quantitative imaging of perfusion using a single subtraction (QUIPSS and QUIPSS II). Magn Reson Med, 1998; 39(5): 702-8

11. Golay X, Stuber M, Pruessmann KP et al: Transfer insensitive labeling technique (TILT): Application to multislice functional perfusion imaging. J Magn Reson Imaging, 1999; 9(3): 454-61

12. Luh WM, Wong EC, Bandettini PA, Hyde JS: QUIPSS II with thinslice TIl periodic saturation: a method for improving accuracy of quantitative perfusion imaging using pulsed arterial spin labeling. Magn Reson Med, 1999; 41(6): 1246-54

13. Petersen ET, Lim T, Golay X: Model-free arterial spin labeling quantification approach for perfusion MRI. Magn Reson Med, 2006; 55(2): 219-32

14. Buxton RB, Frank LR, Wong EC et al: A general kinetic model for quantitative perfusion imaging with arterial spin labeling. Magn Reson Med, 1998; 40(3): 383-96

15. Garcia DM, Duhamel G, Alsop DC: Efficiency of inversion pulses for background suppressed arterial spin labeling. Magn Reson Med, 2005; 54(2): 366-72

16. Lehmann $\mathrm{P}$, Monet $\mathrm{P}$, de Marco $\mathrm{G}$ et al: A comparative study of perfusion measurement in brain tumours at 3 Tesla MR: Arterial spin labeling versus dynamic susceptibility contrast-enhanced MRI. Eur Neurol, 2010; 64(1): 21-26

17. Xu G, Rowley HA, Wu G et al: Reliability and precision of pseudocontinuous arterial spin labeling perfusion MRI on $3.0 \mathrm{~T}$ and comparison with 150-water PET in elderly subjects at risk for Alzheimer's disease. NMR Biomed, 2010; 23(3): 286-93 